BMJ Open

Diabetes

Research

\& Care

\title{
Indirect measure of visceral adiposity 'A Body Shape Index' (ABSI) is associated with arterial stiffness in patients with type 2 diabetes
}

\author{
Ryotaro Bouchi, ${ }^{1}$ Masahiro Asakawa, ${ }^{1}$ Norihiko Ohara, ${ }^{1}$ Yujiro Nakano, ${ }^{1}$ \\ Takato Takeuchi, ${ }^{1}$ Masanori Murakami, ${ }^{1}$ Yuriko Sasahara, ${ }^{1}$ Mitsuyuki Numasawa, ${ }^{1}$ \\ Isao Minami, ${ }^{1}$ Hajime Izumiyama, ${ }^{1,2}$ Koshi Hashimoto, ${ }^{1,3}$ Takanobu Yoshimoto, ${ }^{1}$ \\ Yoshihiro Ogawa ${ }^{1,4}$
}

To cite: Bouchi $\mathrm{R}$,

Asakawa M, Ohara N, et al. Indirect measure of visceral adiposity 'A Body Shape Index' (ABSI) is associated with arterial stiffness in patients with type 2 diabetes. BMJ Open Diabetes Research and Care 2016;4:e00188. doi:10.1136/bmjdrc-2015000188

Received 20 December 2015 Revised 24 February 2016 Accepted 29 February 2016

CrossMark

For numbered affiliations see end of article.

Correspondence to Dr Ryotaro Bouchi; bouchi. mem@tmd.ac.jp

\section{ABSTRACT}

Objective: Among indirect measures of visceral adiposity, A Body Shape Index (ABSI), which is defined as waist circumference (WC)/(body mass index $(\mathrm{BMI})^{2 / 3} \times$ height $^{1 / 2}$ ), is unique in that ABSI is positively correlated with visceral adiposity and is supposed to be independent of BMI. ABSI has been also shown to be linearly and positively associated with visceral fat mass and all-cause and cardiovascular disease (CVD) in the general population. It is, however, uncertain whether ABSI could be associated with arterial stiffness in patients with diabetes.

Methods: This is a cross-sectional study of 607 patients with type 2 diabetes (mean age $64 \pm 12$ years; $40.0 \%$ female). Visceral fat area (VFA, $\mathrm{cm}^{2}$ ) and subcutaneous fat area (SFA, $\mathrm{cm}^{2}$ ) were assessed with a dual-impedance analyzer. In order to estimate the risk for CVD, brachial-ankle pulse wave velocity (baPWV, cm) was used for the assessment of arterial stiffness.

Results: ABSI was significantly and positively correlated with VFA $(r=0.138, p=0.001)$ and negatively associated with BMI $(r=-0.085, p=0.037)$. The correlation of z-score for ABSI with VFA remained significant $(r=0.170, p<0.001)$ but not with $B M I$ $(r=0.009, p=0.820)$. ABSI (standardized $\beta 0.095$, $p=0.043$ ) but not WC (standardized $\beta-0.060$, $p=0.200$ ) was significantly and positively correlated with baPWV in the multivariate model including BMI as a covariate.

Conclusions: $A B S I$ appears to reflect visceral adiposity independently of BMI and to be a substantial marker of arterial stiffening in patients with type 2 diabetes.

\section{INTRODUCTION}

Obesity, especially visceral obesity, has been reported to be associated with insulin resistance, dyslipidemia, and hypertension, thus increasing the risk for cardiovascular disease (CVD). ${ }^{1-4}$ Abdominal visceral fat has been

\section{Key messages}

- A Body Shape Index (ABSI) was significantly and positively correlated with visceral fat area, independently of body mass index.

- Patients with high ABSI were significantly older, with more female predominance, had lower urinary $C$ peptide, longer duration of diabetes, severe diabetic microvascular complications and higher pulse wave velocity (PWV) than those with low ABSI.

- In multivariate linear regression analysis, ABSI remained independently associated with PWV.

strongly associated with cardiovascular risks. ${ }^{56}$ However, a direct measure of visceral fat is limited due to the high cost of imaging procedures such as CT or MRI. On the other hand, several indirect measures of (visceral) adiposity have been developed so far.

Body mass index (BMI) has long been used as a surrogate marker for excess body fat, but its inability to discriminate between fat and lean mass may limit its use to estimate visceral adiposity. ${ }^{7-9}$ Waist circumference (WC) and waist-to-hip ratio (WHR) have alternatively been used as surrogate markers for abdominal obesity and the association of these markers with insulin resistance has been reported to be better than that of BMI. ${ }^{10}{ }^{11}$ While WC is more strongly associated with CVD than BMI, ${ }^{10}$ the J-curve association is observed between BMI or WC and mortality. ${ }^{12-15}$ In addition, the strong correlation between WC and BMI implies that the impact of WC on cardiometabolic risks could be confounded with BMI as are other measures including WHR. A large European cohort study $^{15}$ showed a strong linear association between WC and death after adjusting for BMI, suggesting that both general adiposity (ie, BMI) and visceral 
abdominal adiposity (ie, WC adjusted for BMI) should be simultaneously determined for estimating the risk of death. Eventually, the development of new anthropometric measures has emerged to efficiently estimate both visceral abdominal and general adiposities and precisely predict mortality and cardiovascular events.

In 2012, Krakauer and Krakauer ${ }^{12}$ have proposed a new anthropometric measure (A Body Shape Index, ABSI), which in conjunction with BMI can estimate both visceral abdominal and general overall adiposities. It is important that ABSI is not confounded with BMI (ie, ABSI has been developed to be independent of BMI) for the assessment of abdominal obesity. ABSI, which is adjusted for WC, BMI and height, was able to better predict mortality than WC and BMI in the National Health and Nutrition Examination Survey IV (NHANES IV) population (it is a multiethnic reflecting population in the USA). ${ }^{12}$ Recent studies also demonstrated that ABSI is a robust predictor of all-cause mortality. ${ }^{16} 16$ The largest study from Europe reported that the combination of ABSI and BMI could predict risk for CVD events better than any of the single measures for body composition such as BMI or WC. ${ }^{17}$ Considering the findings in the studies, ${ }^{12} 131617$ it is of importance that ABSI demonstrates a positive linear relationship with allcause or cardiovascular mortality, whereas BMI and WC show the J-shaped associations. ${ }^{14}$ Among patients with diabetes, it is unknown whether ABSI is associated with mortality or cardiovascular events.

Arterial stiffness is a strong predictor of CVD and mortality in the diabetic population, ${ }^{18}{ }^{19}$ and we and others recently reported that increased visceral fat accumulation is associated with arterial stiffness and carotid atherosclerosis, regardless of BMI in patients with diabetes. ${ }^{20}{ }^{21}$ However, little is known regarding the association of ABSI with adiposity and cardiovascular risks in individuals with diabetes. This study was designed to investigate whether ABSI could be associated with arterial stiffness in patients with type 2 diabetes.

\section{METHODS}

Participants

Patients with type 2 diabetes who were admitted to Tokyo Medical and Dental University Hospital for the purpose of glycemic control and/or evaluation of diabetic complications participated in this study. Patients were eligible if they were aged $\geq 20$ years; 607 patients who measured both brachial-ankle pulse wave velocity (baPWV) and visceral fat area (VFA) and subcutaneous fat area (SFA) with a dual bioelectrical impedance analyzer were enrolled. Patients with severe renal impairment (estimated glomerular filtration rate (eGFR) $<15 \mathrm{~mL} / \mathrm{min} / 1.73 \mathrm{~m}^{2}$ or undergoing renal replacement therapy), pregnant women and those with infectious or malignant diseases were excluded. Type 2 diabetes was diagnosed according to the criteria of the Japan Diabetes Society (JDS). ${ }^{22}$ This study complies with the principles laid by the Declaration of Helsinki and has been approved by the ethical committee of Tokyo Medical and Dental University (number 1924).

\section{Clinical and biochemical analysis}

Standardized questionnaires were used to obtain information on smoking, medication, and history. Smoking history was classified as either current smoker or nonsmoker. CVD was defined as the presence of a previous stroke, myocardial infarction, or coronary revascularization procedure. Glycated hemoglobin (HbAlc) was measured by the latex agglutination method. HbAlc levels were expressed in accordance with the National Glycohemoglobin Standardization Programs recommended by the Japanese Diabetes Society. ${ }^{22}$ Urinary albumin and creatinine excretion were measured by the turbidimetric immunoassay and enzymatic method, respectively, in a single $24 \mathrm{~h}$ urine collection. Glomerular filtration rate (GFR) was estimated using the Japanese equation with serum creatinine $(\mathrm{mg} / \mathrm{dL})^{23}$; GFR $=194 \times \mathrm{SCr}^{-1.094} \times \mathrm{age}^{-0.287} \quad($ if female $\left.) \times 0.739\right)$, where SCr stands for serum creatinine in $\mathrm{mg} / \mathrm{dL}$, measured by an enzymatic method. Coefficient of variation of R-R intervals (CV-RR) was used for the assessment of diabetic neuropathy. Height was measured to the nearest $0.1 \mathrm{~cm}$ without shoes using a stadiometer. Weight was measured in light clothing without shoes using a standard scale and recorded to the nearest $0.1 \mathrm{~kg}$. We measured $\mathrm{WC}$ at the umbilical level in minimal respiration in a standing position. BMI was calculated as weight divided by the square of height $(\mathrm{kg} /$ $\mathrm{m}^{2}$ ). ABSI was calculated by the following formula: $\mathrm{ABSI}=\mathrm{WC} / \mathrm{BMI}^{2 / 3} \times$ height $^{1 / 2}{ }^{12}$ VFA and SFA were measured at the level of the umbilicus with a dual bioelectrical impedance analyzer (DUALSCAN, Omron Healthcare Co, Kyoto, Japan).$^{20}$ The baPWV was measured using a volume-plethysmographic apparatus (BP-203RPE II form PWV/ABI, Omron Healthcare Co, Kyoto, Japan), with participants in the supine position after at least 5 min of rest. ${ }^{24}{ }^{25}$ baPWV was calculated as reported previously. ${ }^{26}$ We simultaneously measured baPWV on both the right and left sides and the averaged values from each individual were subjected to statistical analysis.

\section{Statistical analysis}

Statistical analysis was performed using programs available in the SPSS V.21.0 statistical package (SPSS Inc, Chicago, Illinois, USA). Data are presented as mean \pm SD or geometric mean with 95\% CI as appropriate according to data distribution. Differences among the four groups were tested with a one-way analysis of variance (ANOVA) (continuous variables) or $\chi^{2}$ test (categorical variables). Linear regression analyzes with a stepwise procedure were used to assess the cross-sectional association of markers for adiposity including ABSI, BMI, WC, VFA, SFA, and VFA/SFA (V/S) ratio with baPWV. In addition, the correlation of z-scores for these measures was 
determined as reported previously. ${ }^{12}$ The following covariates were incorporated into the analysis: age, gender, duration of diabetes, smoking status, systolic blood pressure, triglycerides, high-density lipoprotein cholesterol, low-density lipoprotein cholesterol, HbA1c, urinary albumin excretion, eGFR, the presence of proliferative diabetic retinopathy and the use of insulin, calcium channel blockers, ACE inhibitors, angiotensin receptor blockers, statins, and antiplatelet agents. Differences were considered to be statistically significant at a $p$ value $<0.05$.

\section{RESULTS}

\section{Clinical characteristics according to ABSI quartiles}

A total of 607 Japanese patients with type 2 diabetes (mean age $64 \pm 12$ years; $40.0 \%$ female) were enrolled in this study. Table 1 shows the clinical characteristics according to the ABSI quartiles among patients with type 2 diabetes. There were significant differences in age, gender, urinary $\mathrm{C}$ peptide, duration of diabetes, albuminuria, CV-RR, and baPWV levels among patients. Patients with high ABSI were significantly older, with more female predominance, had lower urinary C peptide, a longer duration of diabetes, lower CV-RR, and higher baPWV than those with low ABSI. As shown in figure 1, modest increases in VFA, SFA, and WC were observed in parallel with the increase in ABSI among patients with type 2 diabetes. Patients with high ABSI tended to have low BMI compared with those with low ABSI, but the association between ABSI and BMI did not reach statistical significance (figure 1).

\section{Correlation among markers for body composition and baPWV}

Table 2 shows the correlation among anthropometric markers for body composition (height, weight, BMI, WC and $\mathrm{ABSI}$ ), direct measures of visceral and subcutaneous fat (VFA and SFA), and baPWV. ABSI was modestly correlated with WC $(\mathrm{r}=0.391, \mathrm{p}<0.001)$, VFA $(\mathrm{r}=0.138$, $\mathrm{p}=0.001)$ and SFA $(\mathrm{r}=0.161, \mathrm{p}<0.001)$. In contrast, ABSI was negatively correlated to weight $(\mathrm{r}=-0.155, \mathrm{p}<0.001)$ and BMI $(\mathrm{r}=-0.085, \mathrm{p}=0.037)$. We further determined whether the correlation between these markers could be observed after adjusting for age and gender using z-scores for these measures of adiposity. The correlation between z-score for ABSI and VFA was statistically significant $(\mathrm{r}=0.170, \mathrm{p}<0.001)$; however, $\mathrm{z}$-score for ABSI was not associated with $\mathrm{z}$-score for BMI at all $(\mathrm{r}=0.009$, $\mathrm{p}=0.820$ ).

\section{Association between ABSI and baPWV}

Table 3 shows univariate and multivariate linear regression models to investigate the association of anthropometric markers and direct measures of visceral and subcutaneous fat with baPWV. ABSI was significantly and positively associated with increased risk for baPWV in a univariate model. After adjustment for covariates including age, gender, duration of diabetes, and diabetic complications such as nephropathy, ABSI remained to be associated with baPWV. Furthermore, the association between ABSI and baPWV was unchanged even when VFA or BMI was forced into the multivariate model (standardized $\beta 0.101, p=0.047$ and standardized $\beta 0.119$, $\mathrm{p}=0.023$, respectively). Regarding the direct measurement of abdominal fat, SFA was negatively and $\mathrm{V} / \mathrm{S}$ ratio was positively associated with baPWV. Finally, we underwent the sensitivity analysis in which patients treated with pioglitazone $(\mathrm{N}=13)$ were excluded. Finally, the statistical significance between ABSI and baPWV was unchanged (standardized $\beta 0.107, \mathrm{p}=0.041$ ) in the multivariate analysis including age, gender, blood pressure, and diabetic complications and further adjustment for VFA did not attenuate the association (standardized $\beta$ $0.118, \mathrm{p}=0.024)$.

\section{DISCUSSION}

Arterial stiffness is considered to be one of the manifestations of atherosclerosis. It directly reflects the arterial elasticity rather than organic changes in arterial walls such as formation of atherogenic plaques. Increased arterial stiffness has been reported to be strongly associated with mortality and cardiovascular events in patients with diabetes. ${ }^{18} 19$ Therefore, evaluation of arterial stiffness is important to estimate the risk for cardiovascular events among individuals with diabetes. This study, for the first time, demonstrates that ABSI appears to be a substantial marker of arterial stiffening in patients with type 2 diabetes. High ABSI was strongly associated with aging, long duration of diabetes, and low insulin secretion, all of which might account for the association between ABSI and arterial stiffness. In addition, ABSI was modestly and positively correlated to visceral adiposity independently of BMI.

Several previous studies demonstrated the significant association of ABSI with mortality, incident hypertension, diabetes, and CVD. ${ }^{12} 1316{ }^{27-29}$ The strengths of ABSI include the combination of non-invasive measures (WC, height, and weight) and low cost. In this study, consistent with previous studies, ${ }^{10}{ }^{11}$ WC was strongly correlated with BMI ( $r=0.855)$, whereas ABSI was significantly and positively associated with VFA, independently of BMI. These findings suggest that among indirect measures of abdominal adiposity, ABSI has a superiority due to its being independent of BMI, compared with other measures such as WC. In addition, ABSI was significantly associated with pulse wave velocity (PWV) in the multivariate model including BMI as a covariate. Surprisingly, the statistical significance of ABSI with baPWV was not attenuated even after adjusting for VFA, suggesting that the association between ABSI and arterial stiffness might be, at least in part, independent of visceral adiposity. ABSI is a more direct marker of abdominal adiposity than visceral adiposity, since external anthropometrics cannot readily distinguish visceral from subcutaneous 


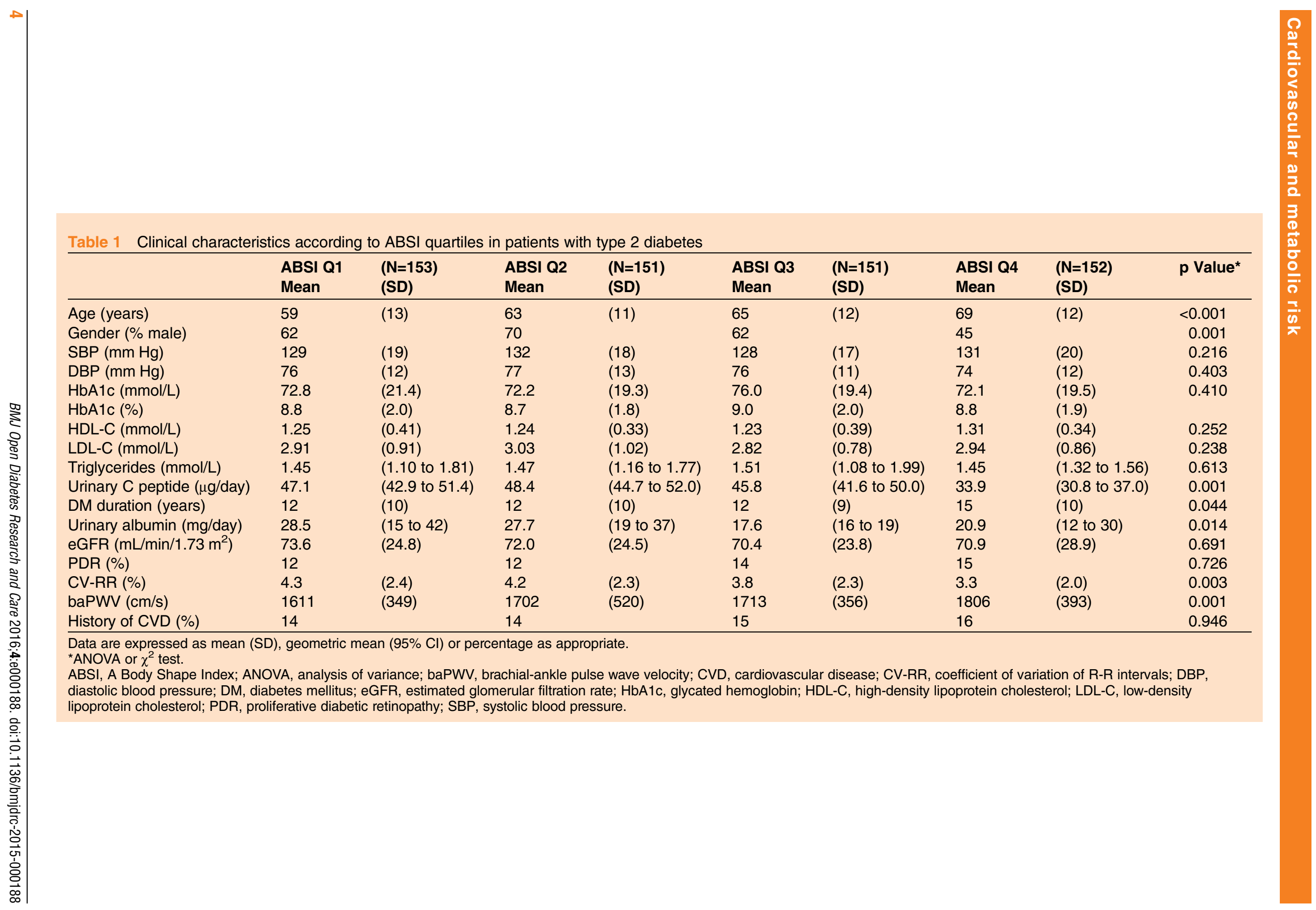




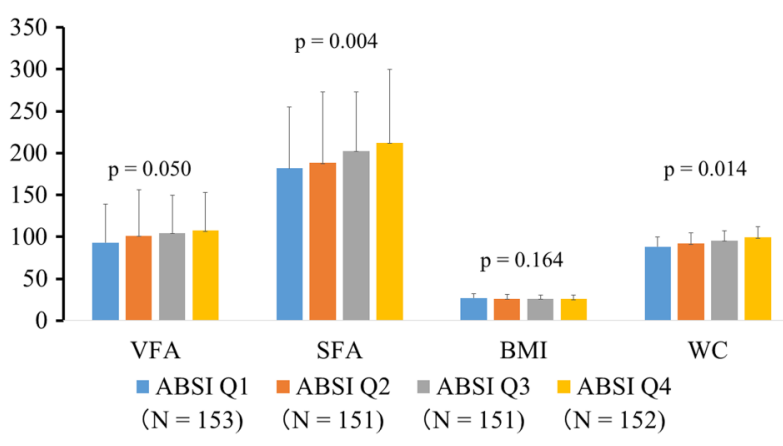

Figure 1 Visceral fat area (VFA, $\mathrm{cm}^{2}$ ), subcutaneous fat area (SFA, $\left.\mathrm{cm}^{2}\right)$, body mass index $\left(\mathrm{BMl}, \mathrm{kg} / \mathrm{m}^{2}\right)$ and waist circumference $(\mathrm{WC}, \mathrm{cm})$ levels according to the quartiles of $A$ Body Shape Index (ABSI) in patients with type 2 diabetes.

$p$ Values by one-way analysis of variance (ANOVA).

fat. This is particularly relevant in the light of our finding that ABSI remained a significant predictor of arterial stiffness even after adjustment for visceral fat. The weak correlation between ABSI and VFA implies the underlying mechanisms other than visceral adiposity which could account for the association between high ABSI and increased arterial stiffness. One possible explanation is that ABSI may inversely reflect muscle mass. Direct evidence has recently been proposed regarding the inverse association between ABSI and fat-free mass ${ }^{30}$; ABSI was negatively, independently of BMI, associated with fat-free mass estimated by bioelectrical impedance analysis (BIA). In addition, ABSI was correlated positively with $\mathrm{C}$ reactive protein and negatively with the reciprocal of insulin, as an index of insulin sensitivity in men. ${ }^{30}$ Also, we and others recently reported that increased visceral fat with normal weight
Table 3 Linear regression analyzes to determine the impact of markers for body composition on baPWV in patients with type 2 diabetes

\begin{tabular}{llrll}
\hline & $\begin{array}{l}\text { Univariate } \\
\text { Standardized } \\
\end{array}$ & \multicolumn{3}{l}{$\begin{array}{l}\text { Multivariate } \\
\text { Standardized }\end{array}$} \\
\hline ABSI & 0.150 & $<0.001$ & 0.095 & p Value \\
WC & -0.138 & 0.010 & -0.060 & 0.043 \\
BMI & -0.128 & 0.008 & -0.151 & 0.200 \\
VFA & -0.086 & 0.100 & -0.059 & 0.001 \\
SFA & -0.182 & $<0.001$ & -0.087 & 0.045 \\
V/S ratio & 0.149 & 0.004 & 0.109 & 0.042 \\
\hline
\end{tabular}

ABSI, A Body Shape Index; baPWV, brachial-ankle pulse wave velocity; BMI, body mass index; SFA, subcutaneous fat area; VFA, visceral fat area; V/S, VFA/SFA; WC, waist circumference.

or normal WC was associated with arterial stiffening ${ }^{20}$ and carotid atherosclerosis. ${ }^{21}$ Together, high ABSI might reflect low skeletal muscle mass with increased visceral abdominal adiposity, systemic microinflammation, and insulin resistance, presumably leading to progression of arterial stiffening, and ABSI could be an important complement to BMI when identifying patients with diabetes at risk for arterial stiffening.

\section{Strengths and limitations}

The strength of our study is that we directly measured VFA and SFA with a dual-impedance analyzer. Eventually, we were able to investigate to what extent ABSI could reflect fat distribution. In addition, this is the first study to show the association between ABSI and arterial stiffness. ABSI was initially developed to predict the risk of death ${ }^{12}$; therefore, it is conceivable that our findings expand the

Table 2 Correlations among markers for body composition

\begin{tabular}{|c|c|c|c|c|c|c|c|}
\hline & Height & Weight & BMI & wc & VFA & SFA & ABSI \\
\hline \multicolumn{8}{|l|}{$r$} \\
\hline Height & 1 & 0.543 & 0.026 & 0.150 & 0.094 & 0.086 & -0.177 \\
\hline Weight & 0.455 & 1 & 0.848 & 0.797 & 0.716 & 0.762 & -0.155 \\
\hline BMI & -0.095 & -0.015 & 1 & 0.855 & 0.795 & 0.846 & -0.085 \\
\hline WC & 0.089 & 0.814 & 0.852 & 1 & 0.803 & 0.856 & 0.391 \\
\hline VFA & 0.050 & 0.771 & 0.771 & 0.763 & 1 & 0.753 & 0.138 \\
\hline SFA & -0.003 & 0.711 & 0.790 & 0.800 & 0.780 & 1 & 0.161 \\
\hline ABSI & -0.073 & -0.015 & 0.009 & 0.482 & 0.170 & 0.226 & 1 \\
\hline \multicolumn{8}{|l|}{$p$ Value } \\
\hline Height & NA & $<0.001$ & 0.501 & $<0.001$ & 0.018 & 0.030 & $<0.001$ \\
\hline Weight & $<0.001$ & NA & $<0.001$ & $<0.001$ & $<0.001$ & $<0.001$ & $<0.001$ \\
\hline BMI & 0.019 & $<0.001$ & NA & $<0.001$ & $<0.001$ & $<0.001$ & 0.037 \\
\hline WC & 0.029 & $<0.001$ & $<0.001$ & NA & $<0.001$ & $<0.001$ & $<0.001$ \\
\hline VFA & 0.216 & $<0.001$ & $<0.001$ & $<0.001$ & NA & $<0.001$ & 0.001 \\
\hline SFA & 0.947 & $<0.001$ & $<0.001$ & $<0.001$ & $<0.001$ & NA & $<0.001$ \\
\hline ABSI & 0.070 & 0.710 & 0.820 & $<0.001$ & $<0.001$ & $<0.001$ & NA \\
\hline
\end{tabular}

Right side (above diagonal) shows correlations of the raw values; left side (below diagonal) shows correlations of the z-scores relative to age-specific and sex-specific means.

ABSI, A Body Shape Index; BMI, body mass index; NA, not applicable; SFA, subcutaneous fat area; VFA, visceral fat area; WC, waist circumference. 
usefulness of ABSI from the prediction of death to risk-estimation of arterial stiffening. This study has a couple of limitations that should be mentioned. First, it is impossible to infer causality because of its cross-sectional design. Second, the population in this study was ethnically and socially homogeneous, because this study was hospital-based and all the patients were Japanese; therefore, generalization of our findings might be limited. It is to be elucidated whether ABSI could be associated with arterial stiffness in populations other than Japanese. Third, we were unable to obtain information on diet and exercise in this study. These lifestyle could affect the distribution of body fat and non-fat mass; thus, exercise and diet may be confounders regarding the association of ABSI with arterial stiffening. Fourth, we could not do the subanalyzes by gender to investigate the association of ABSI with arterial stiffness due to the sample size. Finally, it is to be elucidated whether reduction of ABSI would reduce PWV and cardiovascular events.

In summary, our data suggest that ABSI precisely reflects visceral adiposity independently of BMI and is a substantial marker of arterial stiffening in patients with type 2 diabetes.

\section{Author affiliations}

${ }^{1}$ Department of Molecular Endocrinology and Metabolism, Graduate School of Medical and Dental Sciences, Tokyo Medical and Dental University, Tokyo, Japan

${ }^{2}$ Center for Medical Welfare and Liaison Services, Tokyo Medical and Dental University, Tokyo, Japan

${ }^{3}$ Department of Preemptive Medicine and Metabolism, Graduate School of Medical and Dental Sciences, Tokyo Medical and Dental University, Tokyo, Japan

${ }^{4}$ Department of CREST, Japan Agency for Medical Research and Development, Tokyo, Japan

Acknowledgements The authors thank all the staff of the Department of Molecular Endocrinology and Metabolism, Tokyo Medical and Dental University for their contributions.

Contributors RB designed the study, researched the data, and wrote and edited the manuscript. RB, IM, TY and YO contributed to intellectual discussion and reviewed and edited the manuscript. MN, YS, MA, TT, MM, $\mathrm{YN}, \mathrm{NO}, \mathrm{HI}$ and $\mathrm{KH}$ researched the data. RB is the guarantor.

Funding This work was supported in part by Grants-in-Aid for Scientific Research from the Ministry of Education, Culture, Sports, Science and Technology of Japan.

\section{Competing interests None declared.}

Ethics approval The ethical committee of Tokyo Medical and Dental University. Provenance and peer review Not commissioned; externally peer reviewed.

Data sharing statement No additional data are available.

Open Access This is an Open Access article distributed in accordance with the Creative Commons Attribution Non Commercial (CC BY-NC 4.0) license, which permits others to distribute, remix, adapt, build upon this work noncommercially, and license their derivative works on different terms, provided the original work is properly cited and the use is non-commercial. See: http:// creativecommons.org/licenses/by-nc/4.0/

\section{REFERENCES}

1. Yusuf S, Hawken S, Ounpuu S, et al, INTERHEART Study Investigators. Obesity and the risk of myocardial infarction in 27,000 participants from 52 countries: a case-control study. Lancet 2005;366:1640-9.

2. Fujimoto WY, Bergstrom RW, Boyko EJ, et al. Visceral adiposity and incident coronary heart disease in Japanese-American men. The 10-year follow-up results of the Seattle Japanese-American Community Diabetes Study. Diabetes Care 1999;22:1808-12.

3. Britton KA, Massaro JM, Murabito JM, et al. Body fat distribution, incident cardiovascular disease, cancer, and all-cause mortality. $\mathrm{J}$ Am Coll Cardiol 2013;62:921-5.

4. Kanai H, Matsuzawa $\mathrm{Y}$, Kotani $\mathrm{K}$, et al. Close correlation of intra-abdominal fat accumulation to hypertension in obese women. Hypertension 1990;16:484-90.

5. Després JP, Lemieux S, Lamarche $\mathrm{B}$, et al. The insulin resistance-dyslipidemic syndrome: contribution of visceral obesity and therapeutic implications. Int J Obes Relat Metab Disord 1995;19 (Suppl 1):S76-86

6. Fox CS, Massaro JM, Hoffmann U, et al. Abdominal visceral and subcutaneous adipose tissue compartments: association with metabolic risk factors in the Framingham Heart Study. Circulation 2007;116:39-48.

7. Gómez-Ambrosi J, Silva C, Galofré JC, et al. Body mass index classification misses subjects with increased cardiometabolic risk factors related to elevated adiposity. Int J Obes (Lond) 2011;36:286-94.

8. Okorodudu DO, Jumean MF, Montori VM, et al. Diagnostic performance of body mass index to identify obesity as defined by body adiposity: a systematic review and meta-analysis. Int $J$ Obes (Lond) 2010;34:791-9.

9. Phillips CM, Tierney AC, Perez-Martinez P, et al. Obesity and body fat classification in the metabolic syndrome: impact on cardiometabolic risk metabotype. Obesity (Silver Spring) 2013;21: E154-61.

10. Balkau B, Deanfield JE, Després JP, et al. International Day for the Evaluation of Abdominal Obesity (IDEA): a study of waist circumference, cardiovascular disease, and diabetes mellitus in 168,000 primary care patients in 63 countries. Circulation 2007:116:1942-51.

11. Sumner AE, Sen $S$, Ricks $M$, et al. Determining the waist circumference in African Americans which best predicts insulin resistance. Obesity (Silver Spring) 2008;16:841-84.

12. Krakauer NY, Krakauer JC. A new body shape index predicts mortality hazard independently of body mass index. PLOS ONE 2012;7:e39504.

13. Krakauer NY, Krakauer JC. Dynamic association of mortality hazard with body shape. PLoS ONE 2014;9:e88793.

14. Song X, Jousilahti P, Stehouwer CD, et al, DECODE Study Group. Cardiovascular and all-cause mortality in relation to various anthropometric measures of obesity in Europeans. Nutr Metab Cardiovasc Dis 2015;25:295-304.

15. Pischon $\mathrm{T}$, Boeing $\mathrm{H}$, Hoffmann $\mathrm{K}$, et al. General and abdominal adiposity and risk of death in Europe. $N$ Engl $J$ Med 2008;359:2105-20.

16. Dhana K, Kavousi M, Ikram MA, et al. Body shape index in comparison with other anthropometric measures in prediction of total and cause-specific mortality. J Epidemiol Community Health 2016;70:90-6.

17. Song $X$, Jousilahti $P$, Stehouwer $C D$, et al. Comparison of various surrogate obesity indicators as predictors of cardiovascular mortality in four European populations. Eur J Clin Nutr 2013;67:1298-302.

18. Cruickshank K, Riste L, Anderson SG, et al. Aortic pulse-wave velocity and its relationship to mortality in diabetes and glucose intolerance: an integrated index of vascular function? Circulation 2002;106:2085-90.

19. Mansour AS, Yannoutsos A, Majahalme N, et al. Aortic stiffness and cardiovascular risk in type 2 diabetes. $J$ Hypertens 2013:31:1584-92

20. Bouchi R, Minami I, Ohara N, et al. Impact of increased visceral adiposity with normal weight on the progression of arterial stiffness in Japanese patients with type 2 diabetes. BMJ Open Diabetes Res Care 2015;3:e000081.

21. Kim SK, Park SW, Kim SH, et al. Visceral fat amount is associated with carotid atherosclerosis even in type 2 diabetic men with a normal waist circumference. Int J Obesity (Lond) 2009;33:131-5.

22. Committee of the Japan Diabetes Society on the Diagnostic Criteria of Diabetes Mellitus. Report of the Committee on the Classification and Diagnostic Criteria of Diabetes Mellitus. J Diabetes Investig 2010;1:212-28.

23. Matsuo S, Imai E, Horio M, et al, Collaborators developing the Japanese equation for estimated GFR. Revised equations for estimated GFR from serum creatinine in Japan. Am J Kidney Dis 2009;53:982-92. 
24. Suzuki E, Kashiwagi A, Nishio Y, et al. Increased arterial wall stiffness limits flow volume in the lower extremities in type 2 diabetic patients. Diabetes Care 2001;24 2107-14.

25. Yamashina A, Tomiyama $\mathrm{H}$, Takeda $\mathrm{K}$, et al. Validity, reproducibility, and clinical significance of noninvasive brachial-ankle pulse wave velocity as measures of arterial stiffness. Hypertens Res 2002;25:359-64.

26. Katakami N, Osonoi T, Takahara M, et al. Clinical utility of brachial-ankle pulse wave velocity in the prediction of cardiovascular events in diabetic patients. Cardiovasc Diabetol 2014;13:128.

27. Maessen MF, Eijsvogels TM, Verheggen RJ, et al. Entering a new era of body indices: the feasibility of a body shape index and body roundness index to identify cardiovascular health status. PLOS ONE 2014;9:e107212.

28. Chang Y, Guo X, Chen Y, et al. A body shape index and body roundness index: two new body indices to identify diabetes mellitus among rural populations in northeast China. BMC Public Health 2015;15:794.

29. Fujita M, Sato $\mathrm{Y}$, Nagashima K, et al. Predictive power of a body shape index for development of diabetes, hypertension, and dyslipidemia in Japanese adults: a retrospective cohort study. PLoS ONE 2015;10:e0128972.

30. Biolo G, Di Girolamo FG, Breglia A, et al. Inverse relationship between "a body shape index" (ABSI) and fat-free mass in women and men: insights into mechanisms of sarcopenic obesity. Clin Nutr 2015;34:323-7. 